\title{
Martin Heidegger, profeta de la comunicación indirecta
}

\author{
Martin Heidegger, \\ Prophet of Indirect Comunication
}

\author{
JORGE ROGGERO
}

Universidad de Buenos Aires

Recibido: 30-IX-2014 Aprobado definitivamente: 06-XI-2014

\section{RESUMEN}

La idea de la filosofía sostenida por Heidegger en sus primeros cursos puede ser caracterizada por las notas que Karl Jaspers utiliza para describir a los profetas de la comunicación indirecta. El objetivo de este artículo es mostrar la influencia de la labor metodológica de Søren Kierkegaard en el joven Heidegger.

\author{
PALABRAS CLAVE \\ HEIDEGGER, KIERKEGAARD, COMUNICACIÓN INDIRECTA, \\ INDICACIÓN FORMAL, ÉTICA DEL MÉTODO
}

\begin{abstract}
The idea of philosophy held by Heidegger in his early courses can be characterized by the features that Karl Jaspers uses to describe the prophets of indirect communication. The aim of this article is to show the influence of Søren Kierkegaard's methodological work on the young Heidegger.
\end{abstract}

\author{
KEY WORDS \\ HEIDEGGER, KIERKEGAARD, INDIRECT COMMUNICATION, \\ FORMAL INDICATION, ETHICS OF METHOD
}
(C) Contrastes. Revista Internacional de Filosofia, vol. XX No 2 (2015), pp. 313-329. ISSN: 1136-4076
Licenciatura de Filosofía, Universidad de Málaga, Facultad de Filosofía y Letras Campus de Teatinos, E-29071 Málaga (España)


En PSICOLOGÍA DE LAS CONCEPCIONES DEL MUNDO, Karl Jaspers distingue tres tipos de «maestros proféticos» (prophetische Lehrer) que han influenciado a los hombres con sus doctrinas. Los primeros son los «maestros de determinados principios» (Lehrer bestimmter Prinzipien) que, a partir del «páthos de la verdad encontrada», desarrollan en forma rectilínea no dialéctica principios racionales para la acción. Su posición no es pluralista, consideran como falso todo lo que no se ajusta a sus principios. Exponentes de esta primera actitud son Aristipo, Antístenes, los epicúreos y los estoicos. Los segundos son los «maestros de la totalidad de la vida» (Lehrer der Totalität des Lebens). Se trata de los «grandes formadores de sistemas», tales como Aristóteles, Tomás de Aquino, Hegel; para ellos todo encuentra su lugar, incluso las oposiciones que adquieren un sentido según la posición que ocupan en el ordenamiento de su sistema total. Al respecto, Jaspers señala que sus "envolturas" (Gehäuse) racionales olvidan al individuo en su «existencia viviente y auténtica».

El último tipo de maestros proféticos es el de los «profetas de la comunicación indirecta» (Propheten indirekter Mitteilung), que se caracterizan, paradójicamente, por poner radicalmente en cuestión la tarea del profeta. Su labor se limita a estimular, «llamar la atención» (aufmerksam machen), propiciar un estado de intranquilidad, volver las cosas problemáticas, «pero no dan prescripciones, no enseñan cómo hay que vivir; [son] aquellos que en apariencia desgarran, llevan a la desesperación, crean dificultades y, sin embargo, no ofrecen nada "positivo". [...] Los filósofos de la comunicación indirecta se impulsan interiormente, como individuos, hacia el hombre individual; apelan a la vida que hay en el otro, al que ayudan a crecer estimulándolo y desarrollando el médium de la reflexión infinita; crecimiento que ellos, sin embargo, no quieren dar por sí mismos como doctrina imperativa. Si se les expone el deseo de ser sus discípulos, lo rechazan; aman la libertad en el otro. [...] Representativos para este tipo son Sócrates, Kant, Kierkegaard». ${ }^{1}$

Jaspers considera a Kierkegaard el máximo exponente de este profetismo filosófico ${ }^{2}$ que recurre a la comunicación indirecta. A partir de las sugerencias

$1 \quad$ Ib., pp. 376-377.

$2 \mathrm{Al}$ comienzo de su libro, Jaspers afirma que «la filosofía ha sido desde siempre algo más que contemplación universal, ella dio impulsos, erigió cuadros de valores, dio a la vida del hombre sentido y meta, le dio el mundo en el que se sintió a salvo, en una palabra: le dio, concepción del mundo [Weltanschauung]. [...] Los filósofos no eran solamente observadores pacíficos, irresponsables, sino también impulsores y formadores del mundo. A esta clase de filosofía la llamamos filosofía profética [prophetische Philosophie]». Ib., p. 2. 
de Otto Pöggeler ${ }^{3}$ y John van Buren ${ }^{4}$ respecto de los «impulsos» $\rangle^{5}$ de Kierkegaard en el trabajo desarrollado por el joven Martin Heidegger en sus primeros cursos en la Universidad de Friburgo (1919-1923) y, en particular, de su presencia en el trabajo metodológico, ${ }^{6}$ sostendré que la filosofía heideggeriana de ese período reúne las características con las que Jaspers describe a los profetas de la comunicación indirecta. Si se tiene en cuenta que desde 1911, Heidegger leía las traducciones de Kierkegaard hechas por Theodor Haecker para Der Brenner, y que entre 1919 y 1921, escribió una reseña del libro de Jaspers en la que se extendió en el análisis de ideas kierkegaardianas ${ }^{8}$ no parece tan aventurada una afirmación en este sentido. ${ }^{9}$ La reflexión metodológica del joven Heidegger está signada por una particular presencia de la comunicación indirecta kierkegaardiana. La radicalización de la fenomenología llevada a cabo por Heidegger

3 Cf. O. Pöggeler, «Destruction and Moment», trad. D. Magurshak, en Th. Kisiel y J. van Buren (eds.), Reading Heidegger from the start: essays in his earlier thought. Albany: State University of New York Press, 1994, pp. 140-144.

4 Cf. J. van Buren, The young Heidegger. Rumor of the hidden King. Bloomington: Indiana University Press, 1994, pp. 166-167, 169, 182, 189, 195, 366, 376, 380.

5 En 1923, en el último curso dictado en Friburgo, antes de su partida a Marburgo, Heidegger reconoce: «Compañero en la búsqueda fue el joven Lutero y modelo Aristóteles, a quien aquél odiaba. Impulsos [Stöße] me dió Kierkegaard y los ojos me los colocó Husserl». M. Heidegger, Gesamtausgabe. II. Abteilung: Vorlesungen 1919-1944. Band 63. Ontologie. Hermeneutik der Faktizität. Frankfurt am Main: Vittorio Klostermann, 1988, p. 5. En adelante, me referiré a los textos de Heidegger con las siglas GA (Gesamtausgabe) seguidas del correspondiente número de tomo.

6 Cf. J. van Buren, The young Heidegger.., op. cit., pp. 326-328, 339-341, 353, 388.

7 Cf. Th. Kisiel, «Heidegger's Apology: Biography as Philosophy and Ideology», en Th. Kisiel, Heidegger's way of thought. London: Continuum, 2002, p. 26. Para un listado completo de las traducciones al alemán de la obra de Kierkegaard Cf. H. Schulz, «Germany and Austria: a modest head start», en J. Stewart (ed), Kierkegaard Research: sources, receptions and resources. Volume 8. Kierkegaard's International Reception. Tome I: Northern and Western Europe. Farnham: Ashgate Publishing, 2009, pp. 388-420.

8 GA 9, p. 1-44.

9 Luego de la aparición de los textos de Pöggeler y van Buren, que retoman la interpretación de Gadamer de la indicación formal como un kierkegaardiano «llamar la atención» (aufmerksam machen) (Cf. H.-G. Gadamer, «Die religiöse Dimension (1981)», en H.-G. Gadamer, Gesammelte Werke, Band 3: Neuere Philosophie: 1. Hegel, Husserl, Heidegger. Tübingen: J. C. B. Mohr Verlag, 1987, p. 316), la influencia de la comunicación indirecta en el joven Heidegger es una cuestión aceptada ya por muchos comentaristas. Cf. J. Adrián Escudero, «Heidegger y la indicación formal: hacia una articulación categorial de la vida humana», Diánoia, vol XLIX nro. 52 (mayo 2004), pp. 29-32; S. Mcgrath, The early Heidegger \& medieval philosophy. Phenomenology for the Godforsaken. Washington D.C.: The Catholic University of America Press, 2006, pp. 51-53; B. Crowe, Heidegger's religious origins. Destruction and authenticity. Bloomington: Indiana University Press, 2006, p. 77, entre otros. 
implica una revisión de la conceptualización filosófica que tiene por resultado la indicación formal (formale Anzeige). En ella no sólo pueden observarse los puntos en común con la metodología indirecta de Kierkegaard, sino que también puede sostenerse que ambos autores comparten, de alguna manera, ciertos «presupuestos». El proyecto filosófico del joven Heidegger constituye una «ética del método». Su reformulación metodológica se asienta sobre ciertos supuestos que permiten articular una ética de respeto radical por la alteridad.

A fin de demostrar estas afirmaciones, en un primer apartado expondré la idea de la comunicación indirecta en la obra de Kierkegaard. En el segundo me detendré en la propuesta filosófica de Heidegger en estos cursos tempranos, analizando particularmente el planteo metodológico centrado en la indicación formal y su relación con la comunicación indirecta. En el último apartado esbozaré algunas conclusiones considerando al joven Heidegger como un profeta de la comunicación indirecta.

\section{LA COMUNICACIÓN INDIRECTA KIERKEGAARDIANA}

Luis Guerrero sostiene que existen ciertos supuestos -que, en rigor, son consecuencias que se desprenden de las características mismas de la existencia- que permiten advertir la necesidad de un método indirecto y justificar el uso de los pseudónimos. ${ }^{10}$ El primero de estos supuestos es la afirmación de una verdad subjetiva. Kierkegaard entiende que existen dos tipos de verdades: las objetivas, que son las propias del conocimiento científico y que su descubrimiento no implican un compromiso vital con ellas; y las subjetivas, que no pueden alcanzarse más que a través de un apropiación que exige tomar postura. Se trata de las verdades que involucran la existencia y que sólo pueden comprenderse a través de ese movimiento de apropiación. ${ }^{11}$ Con esta distinción, Kierkegaard quiere destacar la relación existencial a la que nos compromete la verdad subjetiva. En una anotación en su Diario, de agosto de 1835, ya se encuentra esta misma idea: «Lo que realmente necesito es tener en claro qué debo hacer y no qué debo saber [...] De lo que se trata es de entender mi destino, de comprender que es lo que Dios realmente quiere que haga: la cuestión es

10 Cf. L. Guerrero, La verdad subjetiva. Søren Kierkegaard como escritor. México: Universidad Iberoamericana, 2004, pp. 29-43. Me detengo exclusivamente en los tres primeros porque son los que se relacionan más directamente con su concepción de la existencia humana, que es el problema que también preocupa a Heidegger.

11 Cf. S. Kierkegaard, Søren Kierkegaards Skrifter. Bind 7. Afsluttende uvidenskabelig Efterskrift, København, Gads Forlag, 2002, p. 182. En adelante, utilizaré esta edición de la obra completa de Kierkegaard y me referiré a ella con las siglas SKS (Søren Kierkegaards Skrifter), agregando el número de tomo y el nombre de la obra en cada caso. 
encontrar una verdad que sea verdad para mí, encontrar una idea por la cuál esté dispuesto a vivir y morir». ${ }^{12}$ Esta tarea de apropiación de una «verdad que sea verdad para mí» es absolutamente individual y por eso se requiere de una comunicación indirecta para señalar su importancia. No hay manera de transmitir objetivamente una verdad que se define como «incertidumbre objetiva apropiada firmemente por la más apasionada interioridad». ${ }^{13}$

El segundo supuesto es el de la libertad. Kierkegaard postula tres estadios de la existencia: estético, ético y religioso. La existencia estética es la que vive bajo las categorías de la sensibilidad y tiene al goce como su único cometido. El primer volumen de $O$ lo uno o lo otro está dedicado a la descripción de este tipo de existencia. El esteta («A») vive en la inmediatez, «es lo que es», se abstiene de tomar una decisión porque considera que elija lo uno o lo otro lo va a lamentar. «A» se sitúa en una esfera en la que el principio de contradicción, la negación hegeliana, aún no tiene aplicación. No tomando decisiones no tiene que lidiar con las consecuencias negativas. Ahora bien, el principio de contradicción es el que rige en la realidad, en la actualización, «A» permanece en el plano de la posibilidad. «A» entiende que vivir una existencia aeterno modo no consiste en intentar alcanzar una síntesis que pueda mediar entre las oposiciones que surgen luego de elegir entre lo uno o lo otro, sino que la verdadera eternidad yace en permanecer en un estadio anterior a la oposición aut-aut.

La existencia ética comienza, precisamente, cuando se toma una decisión por lo uno o lo otro. Se trata del plano en el que uno «deviene lo que deviene» por medio de la elección. En sentido estricto, la elección estética no es una verdadera decisión, sólo la elección ética nos enfrenta a una decisión «absoluta», una decisión que involucra nuestra mismidad. Kierkegaard no concibe el «sí mismo» como una sustancia que subyace sino como una tarea a realizar, algo que debemos alcanzar por medio de nuestra elección. El Juez William, personaje que representa el discurso ético en el segundo volumen de $O$ lo uno o lo otro, trata de convencer al esteta de que elija elegir. Sólo a través de la decisión puedo constituirme como una mismidad.

$\mathrm{Y}$, sin embargo, la decisión ética no es la decisión final. En el «Ultimatum», el pastor de Jutland afirma que nadie es justo ante Dios, la ética es orgullo ante Dios. ${ }^{14}$ Es necesario un pasaje del estadio ético al estadio religioso. En La enfermedad mortal, el pseudónimo Anti-Climacus sostiene la necesidad de atravesar la desesperación para acceder al estadio religioso. El tener conciencia de esta desesperación -que todos padecemos: «no hay ni siquiera uno solo

12 SKS 17, Journalen $A A-B B-C C-D D$, p. 24

13 SKS 7, Afsluttende uvidenskabelig Efterskrift, p. 186.

14 Cf. SKS 3, Enten-Eller. Anden del, pp. 317-332. 
que no sea un poco desesperado»- ${ }^{15}$ es un paso necesario para alcanzar la fe que nos libera de la desesperación. Por medio de la fe, «al autorrelacionarse y querer ser sí mismo, el yo se apoya de una manera lúcida en el Poder que lo ha creado»: ${ }^{16}$ se apoya en Dios. Todo lo que no procede de la fe lleva a la desesperación porque procede del pecado. La existencia religiosa responde a la fe, implica el «salto de la fe», el salto hacia el absurdo, salto que nos expone a la inaudita paradoja de la fe, «paradoja que no puede reducirse a ningún razonamiento; porque la fe comienza precisamente donde acaba la razón». ${ }^{17} \mathrm{La}$ fe se encuentra por encima de la razón, corresponde a un orden irreductible a la racionalidad. La existencia religiosa implica la negación de lo general. «La fe es esa paradoja según la cual el Individuo está por encima de lo general y siempre de tal manera, cosa importante, el movimiento se repite y como consecuencia el Individuo, luego de haber estado en lo general se aísla en lo sucesivo como Individuo por encima de lo general»». ${ }^{18} \mathrm{El}$ individuo religioso de Temor y temblor reconoce la existencia de una legalidad interior más elevada que la normatividad de lo general.

Es importante advertir que ninguno de estos estadios implica una evolución necesaria respecto a otro, una evolución que la humanidad llevaría a cabo a través de la historia; sino que la permanencia en uno o en otro depende exclusivamente de la libertad individual. Asumir el compromiso existencial que implica esta libertad es una tarea que debe ser llevada a cabo individualmente. Y justamente es el respeto por esa libertad lo que obliga a asumir una metodología indirecta para transmitir esta convicción. Tanto los dos volumenes de $O$ lo uno o lo otro como La enfermedad mortal o Temor y temblor son firmados por un pseudónimo. De esta forma, Kierkegaard busca ser «invisible». Lo importante es la reflexión absolutamente individual que el lector pueda emprender a partir de la lectura de los textos, por eso Kierkegaard utiliza los pseudónimos, porque el autor no debe ser una distracción ni debe intentar transmitir con una argumentación directa la tarea que el lector debe realizar en su fuero más íntimo. ${ }^{19}$

El tercer supuesto es el de la existencia cristiana. Kierkegaard entiende que sólo la existencia religiosa puede fundar una existencia auténtica; únicamente ante Dios y reconociendo su dependencia respecto de Él, el individuo puede devenir un sí mismo. «Porque, cabalmente, la fórmula que describe la situación del yo una vez que ha quedado exterminada por completo la desesperación es

15 SKS 11, Sygdommen til Døden, p. 138.

16 Ib., p. 130.

17 SKS 4, Frygt og Baeven, p. 147.

18 Ib., p. 149.

19 Cf. SKS 7, "En første og sidste Forklaring", Afsluttende uvidenskabelig Efterskrift, p. 569. 
la siguiente: que al autorrelacionarse y querer ser sí mismo, el yo se apoya de una manera lúcida en el Poder que lo ha creado». ${ }^{20}$

Kierkegaard se propone transmitir la verdad de que la verdad debe ser buscada individualmente y no puede ser alcanzada teóricamente, sino que exige una apropiación que comprometa toda nuestra existencia. La importancia de esta tarea exige una metodología de comunicación indirecta que entre en tensión con una comunicación directa; pues no se trata de transmitir un contenido objetivo, sino de indicar una actitud que debe ser asumida individualmente. Así, la comunicación kierkegaardiana, «lo mismo que el milagro, solamente puede servir para hacer caer en la cuenta, y así que el que acaba de ser suscitado a la atención, chocando con la contradicción, pueda elegir creer o no». ${ }^{21}$

\section{LA COMUNICACIÓN INDIRECTA HEIDEGGERIANA}

En los primeros cursos de Heidegger la preocupación metodología es recurrente. Basta recordar las palabras de la famosa lección dictada en el semestre de emergencia de guerra (Kriegsnotsemester) en 1919: «nos hallamos ante una encrucijada metodológica [methodische Wegkreuzung] que decide sobre la vida o la muerte de la filosofía en general. Nos hallamos ante un abismo en el que, o [entweder] bien nos precipitamos en la nada -es decir, en la nada de la objetividad absoluta - o [oder] bien logramos el salto [Sprung] a otro mundo o, más precisamente, en realidad tan sólo al mundo». ${ }^{22}$ La decisión sobre el método es una decisión en la que se nos juega la vida. La filosofía no es un mero ejercicio teórico, sino más bien un modo de vida ${ }^{23}$ que nos conmina a asumir un compromiso existencial. Lo que se debate en la «encrucijada metodológica» no es una cuestión secundaria, sino la elección entre dos maneras de hacer filosofía que conllevan dos modos de vida radicalmente opuestos. Pero ¿en que consiste esta relación entre vida y método que hace de la reflexión metodológica algo primordial? La respuesta -que se insinúa en el lenguaje kierkegaardiano del pasaje- debe buscarse en la reseña del libro de Jaspers que Heidegger comienza a escribir ese mismo año. La comunicación indirecta de Kierkegaard se constituye en modelo de la inescindible imbricación entre método y vida. «Respecto a Kierkegaard, debo señalar que no a menudo, en la filosofía o la teología, [...] es alcanzado un nivel tan riguroso de conciencia metodológica como la suya.

20 SKS 11, Sygdommen til Døden, p. 130.

21 SKS 12, Indøvelse i Christendom, p. 139.

22 GA 56/57, p. 63.

23 "[La investigación filosófica] misma constituye un determinado cómo de la vida fáctica y, como tal, co-temporiza en su realización el ser concreto de la vida en sí misma en cada ocasión y no solamente en una posterior aplicación." GA 62, p. 351. 
Se renuncia a lo decisivo en Kierkegaard, si se pasa por alto esta conciencia metodológica o es tomada como algo de importancia secundaria»». ${ }^{24}$

La co-implicación de la comunicación indirecta con la reflexión sobre la existencia pone al descubierto que el método no puede consistir en una estrategia que se imponga arbitraria y externamente, sino que son las características mismas del fenómeno (de la existencia, de la vida) las que lo determinan. Son los rasgos éticos y religiosos de la existencia los que demandan una comunicación indirecta. En el citado pasaje del curso de 1919, Heidegger señala que la preeminencia de lo teórico en la historia de la filosofía nos arroja a la nada pues no es posible asir la cosa misma si se la tergiversa en una conceptualización teórica. El «pecado mortal» (Todsünde) contra la fenomenología consiste precisamente en «construir un método desde arriba o desde afuera» ${ }^{25}$, es decir, en no tomar lo que se da tal como se da, según prescribe el «principio de todos los principios» formulado por Husserl en el $\S 24$ de Ideas para una fenomenología pura y una filosofía fenomenológica. ${ }^{26}$ La fenomenología, la filosofía, debe dar el salto, debe ser radicalizada en dirección al hic et nunc de la existencia humana, a su facticidad. Sólo si se elige este camino es posible no sucumbir ante la desvivificación (Entlebung), designificación (Entdeutung) y deshistorización (Entgeschichtligung) ${ }^{27}$ del enfoque teórico.

«La filosofía tiene por tarea sostener la facticidad de la vida y fortalecer la facticidad de la existencia». ${ }^{28}$ Ahora bien, esta tarea sólo puede llevarse a cabo a través de la aceptación del carácter móvil, inestable, de la vida, y de la consecuente dificultad que esto implica. Es más, reconocer la movilidad (Bewegtheit) de la vida conlleva advertir su tendencia a la caída (Verfallen), a la ruinancia (Ruinanz) ${ }^{29}$ Esta tendencia no es otra cosa que la propensión de la vida a ocultar su carácter fáctico. Preservar la facticidad de la vida demanda una tarea crítica-destructiva de esta comprensión habitual en la que la vida se nos manifiesta. Esta labor debe ser llevada a cabo originariamente, a partir de un motivo que surge de la vida misma sin tergiversar sus propios términos. ${ }^{30}$

En el semestre de invierno de 1920/1921, Heidegger dicta un curso sobre el apóstol Pablo. La experiencia de la vida fáctica de las primeras comunidades

24 GA 9, p. 41.

25 GA 56/57, p. 110.

26 Cf. E. Husserl, Husserliana. Edmund Husserl Gesammelte Werke. Band III/1. Ideen zu einer reinen Phaenomenologie und phaenomenologischen Philosophie. Erstes Buch. Den Haag: Martinus Nijhoff, 1976, p. 51. En adelante, me referiré a los textos de Heidegger con las siglas Hua (Husserliana) seguidas del correspondiente número de tomo.

27 Cf. GA 56/57, p. 89.

28 GA 59, p. 174.

29 GA 61, p. 131.

30 Volveré sobre esta cuestión en el apartado siguiente. 
cristianas a las que Pablo se dirige en sus epístolas se constituye en modelo de preservación de la facticidad. El fenómeno de la espera de la parusía da cuenta de una experiencia de la temporalidad originaria. El cristiano primigenio acepta la dificultad, la «continua inseguridad» (ständige Unsicherheit), la «absoluta tribulación» (absolute Bedrängnis) que implica vivir en la espera del Mesías. ${ }^{31}$ El creyente acepta que debe vivir sobrio y en vigilia pues «el día del Señor llega como un ladrón por la noche». ${ }^{32}$ Esta vida fáctica cristiana es caracterizada por Heidegger como el producto de una transformación que afecta el cómo del comportarse, es decir, el sentido de la realización (Vollzugssinn) de la vida fáctica, pero no implica ningún cambio respecto del contenido: la facticidad es vivida en la forma del hos mé (como no), ${ }^{33}$ es decir, como un comportamiento que transforma el complejo realizativo (Vollzugszusammenhang) pero no el objetivo (Objektzusammenhang).

En sus primeros cursos, Heidegger trabajaba con una partición de la intencionalidad en tres sentidos. El fenómeno puede ser caracterizado según un sentido de contenido (Gehaltssinn), un sentido de referencia (Bezugssinn) $\mathrm{y}$ un sentido de realización (Vollzugssinn) ${ }^{34} \mathrm{El}$ sentido de contenido responde al «qué», el sentido de referencia responde al «cómo» en el que el fenómeno se da, y el sentido de realización responde al «cómo» en el que el sentido de referencia se realiza. La intencionalidad de la vida fáctica pone el acento en los dos últimos sentidos. La facticidad bajo el signo del hos mé demanda una metodología que pueda dar cuenta de ella sin desvivificarla, sin designificarla y sin deshistorizarla. Heidegger se enfrenta a una cuestión similar a la que preocupa a Kierkegaard: la vida fáctica como la existencia religiosa no pueden ser objetivadas. Pero, entonces, ¿cómo es posible aprehenderla? Y en tal caso, ¿cómo puede transmitirse esa aprehensión sin desvirtuar su fluir pre-teórico en una configuración conceptual fija? Estos problemas serán caracterizados por Heidegger como los problemas de la intuición (Anschauung) y de la expresión (Ausdruck), y lo llevarán a postular la necesidad de una «teoría de la formación filosófica del concepto» (Theorie der philosophischen Begriffsbildung). ${ }^{35}$ Dar cuenta de la vida fáctica, en sus propios términos, implica desarrollar algún tipo de estrategia indirecta, algún tipo de herramienta conceptual que opere como un simple «llamar la atención» (aufmerksam machen), como indicador de una

31 GA 60, p. 105 y p. 97.

321 Tes $5,2$.

33 «Os digo, pues, hermanos, el tiempo es corto; por lo demás, que los que tienen mujer vivan como no teniéndola y los que lloran como no llorando, y los que están alegres como no estándolo; los que compran como no poseyendo, y los que disfrutan del mundo como no abusando de él. Pasa de hecho la apariencia de este mundo». 1 Cor 7, 29-33.

34 Cf. GA 58, p. 261, GA 60, p. 63.

35 Cf. GA 59. 
dirección que no puede ser objetivada. Esta herramienta será la «indicación formal» (formale Anzeige). «En la metodología llamamos "indicación formal" al empleo de un sentido que guía la explicación fenomenológica. [...] [que] si bien orienta la consideración, no introduce ninguna opinión prejuzgadora en los problemas». ${ }^{36}$

Según Günther Stern, ${ }^{37}$ asistente a los primeros cursos de Heidegger, es probable que el término «indicación» (Anzeige) refiera a la distinción entre expresiones objetivas (objektive Ausdrücke) y expresiones ocasionales (okkasionelle Ausdrücke) propuesta por Husserl en la Primera Investigación Lógica. Las expresiones objetivas son aquellas que por tener un significado objetivo son comprendidas sin necesidad de atender a quién las manifiesta y las circunstancias en que son dichas. Ejemplo de este tipo de expresiones son las utilizadas para principios y teorías de las ciencias «abstractas».

Las expresiones ocasionales, por el contrario, son aquellas que sólo adquieren significación en relación con las «efectivas circunstancias de la manifestación» y con quien las emite. Husserl afirma que se trata de las expresiones que «sirven a las necesidades prácticas de la vida común»». ${ }^{38} \mathrm{Y}$ unos reglones más adelante, da un ejemplo. «Toda expresión que contenga un pronombre personal, carece de sentido objetivo. La palabra "yo" nombra en cada caso una persona distinta y lo hace mediante una significación siempre nueva. Cuál sea su significado en cada caso, solo puede ser tomado del discurso viviente y su pertenencia a circunstancias intuitivas». ${ }^{39}$ La palabra «yo» tiene una particular función indicativa (Anzeigende Funktion): reenvía el discurso a quien lo está emitiendo, «dice al oyente: tu interlocutor se mienta a sí mismo» ${ }^{40} \mathrm{y}$, de esta forma, superpone dos tipos de significaciones. Por un lado, la significación indicativa (anzeigende Bedeutung) que refiere a la función general vacía del «yo»; por el otro, la significación indicada (angezeigte Bedeutung) que remite al yo singular y concreto en su aquí y ahora. En la Sexta Investigación, Husserl insiste en la importancia de la indicación. Cuando el oyente escucha «yo»o «esto» o «aquí», entiende cierta generalidad, sabe que se está indicando algo, pero «el verdadero objetivo de la expresión no reside en este universal, sino en la intención directa hacia el objeto respectivo»». ${ }^{41} \mathrm{El}$ oyente necesita tener una intuición directa de lo mentado por el hablante para comprender lo expresado. Heidegger toma este carácter de la indicación que necesita ser apropiada, ser re-

36 GA 60, p. 55.

37 Citado por John van Buren. Cf. J. van Buren, The young Heidegger..., op. cit., pp. 328 y 406 n. 5.

38 Hua XIX, p. 87.

39 Id.

40 Ib., p. 88.

$41 \quad$ Ib., p. 558. 
ferida a circunstancias concretas, a un aquí y ahora, para adquirir significación.

Con el adjetivo «formal» (formal), Heidegger alude a la distinción husserliana de dos tipos de universalización: la generalización (Generalisierung) y la formalización (Formalisierung). La generalización es la universalización de acuerdo al género y a la especie. Por ejemplo, rojo es un color, color es una cualidad sensible. En estos pasajes de niveles de generalización se da una continuidad asegurada por una limitación a un sector temático en base a un contenido. Ahora bien, el tránsito que va de la «cualidad sensible» (sinnliche Qualität) a la «esencia» (Wesen) o de «esencia» a «objeto» (Gegenstand) implica una fractura en la delimitación del sector temático. La «esencia» no sólo no tiene un lugar asignado en la secuencia de niveles, sino que puede predicarse de cualquiera de ellos. La formalización, a diferencia de la generalización, no está sujeta a un sector temático ni a una secuencia de niveles. Ella no surge de un contenido objetivo sino de la referencia actitudinal (Einstellungsbezug) misma. ${ }^{42}$ En este sentido, la formalización no prejuzga pues sólo da cuenta del cómo del darse sin referir a un qué. Sin embargo, a partir del curso sobre Pablo, Heidegger va a considerar que la formalización husserliana aún contiene la limitación de encontrarse en el campo actitudinal teórico. La formalización husserliana predetermina el cómo del darse del fenómeno desde cierta noción de lo «general» (allgemein), propia de la teoría. ${ }^{43}$ Es necesario radicalizar la idea husserliana de formalización. La indicación formal heideggeriana no es un tipo de universalización, pues orienta la explicación fenomenológica de tal manera que ésta sea determinada por el fenómeno mismo y no por algún tipo de actitud teórica ideal. Tanto la generalización como la formalización husserlianas están teóricamente motivadas y, por lo tanto, constituyen algún tipo de imposición «desde afuera» a los términos en que el fenómeno se manifiesta. Ambas buscan el orden, de manera directa en la generalización, de manera indirecta en la formalización. La indicación formal, por el contrario, no está preocupada por el orden, sino más bien por liberarse de toda disposición o clasificación, permaneciendo abierta e indeterminada.

La indicación formal es la herramienta conceptual que, alejándose del sentido de contenido enfatizado por las teorías tradicionales, permite aprehender y expresar los sentidos de referencia y de realización de la vida fáctica. En esta posibilidad está su originariedad, en su carácter «formal» que se limita a indicar un cómo, una forma, una manera de darse del fenómeno. Ahora bien,

42 Cf. GA 60, p. 61.

43 Cf. Ib., p. 59. En el curso del semestre de guerra, en un intento por encontrar una forma de expresión que no caiga en la desvivificación teórica, Heidegger ya se había detenido en el procedimiento de formalización y aún lo consideraba una alternativa plausible. Cf. GA 56/57, pp. 112-115. 
en su carácter de «indicación», la indicación formal tiene un carácter radicalmente negativo, no sólo rechaza todo contenido sino que también previene de cualquier reenvío a un sector temático a través de una predeterminación teórica de un cómo del darse del fenómeno. ${ }^{44}$ El sentido de referencia queda suspendido pues la plenitud de sentido sólo puede alcanzarse a través del sentido de realización en el que el intérprete es reenviado a la experiencia misma del fenómeno que la indicación formal señala. Como bien destaca John D. Caputo, las «indicaciones formales» son pues «principios fáctico-históricos cuyo contenido real está siempre concretamente por ser determinado por la manera en que el principio es apropiado»..$^{45}$

En la reseña al libro de Jaspers, Heidegger refiere al planteamiento del problema de la existencia mediante una indicación formal: «"Existencia” [...] puede ser entendida como un modo determinado del ser, como un determinado sentido del "es", que esencialmente "es" el sentido del (yo) "soy", que no se obtiene genuinamente en la opinión teórica, sino en el cumplimiento activo del "soy", un modo de ser del ser del "yo". El ser del sí mismo [Selbst], así entendido, quiere decir, bajo su indicación formal, existencia. Con esto ya se da a entender de dónde se debe extraer el sentido de la existencia en cuanto determinado Cómo o modalidad del sí mismo (del yo). Así pues, lo decisivo es que yo me tenga, esto es, la experiencia fundamental en la que yo me encuentro a mí mismo como sí mismo, de tal manera que al vivir esa experiencia, y de acuerdo con su sentido, yo puedo preguntar el sentido de mi "yo soy"». ${ }^{46}$ Más allá de que Heidegger considere que Kierkegaard cae acríticamente en una determinada concepción de la existencia, sin lograr un acceso a su sentido genuino ${ }^{47}$ este pasaje deja en claro la deuda que el joven alemán tiene con el danés. Heidegger no sólo comparte con Kierkegaard la preocupación por el sí mismo como problemática esencial de la filosofía («pues en última instancia, de lo que se trata la filosofía es de dicho sí mismo $[$ Selbst $\left.]\rangle^{48}\right)$, sino que ésta también lo lleva a una consideración de la extrema importancia de la cuestión metodológica. Si el sentido de la existencia «no se obtiene genuinamente en la opinión teórica, sino en el cumplimiento activo del "soy"», entonces es necesario pensar un método que identifique como problema principal el «cómo del planteamiento del cumplimiento activo de la apropiación [Aneignung]». ${ }^{49}$ Este es el propósito final de la indicación formal. Se trata de encontrar la manera de

44 Cf. GA 60, p. 63-64.

45 J. D. Caputo, «Heidegger's Kampf», en J. D. Caputo, Demythologizing Heidegger. Bloomington: Indiana University Press, 1993, p. 43.

46 GA 9, p. 29.

47 Cf. Ib., p. 11.

48 Ib., p. 35.

49 Id. 
«llamar la atención» (aufmerksam machen) respecto a una dirección que debe ser experimentada de manera absolutamente individual. Al modo de la comunicación indirecta kierkegaardiana, el sentido de realización (Vollzugssinn) de la indicación formal reenvía al intérprete a la experiencia misma del fenómeno. El sentido último reside, precisamente, en esa apropiación. Kierkegaard diría: «...la verdad es la interioridad, objetivamente no existe la verdad, sino que es en la misma apropiación en lo que consiste la verdad». ${ }^{50}$

\section{Martin HeIDEgGer, PROFETA DE LA COMUNICACIÓN INDIRECTA}

Profeta es quien procura transmitir una revelación divina que le ha sido confiada por medio de una experiencia personal con Dios. Su labor es hermenéutica ya que a través de la profecía el profeta interpreta la historia. Todos estos elementos se encuentran en el joven Heidegger. ${ }^{51}$ En primer lugar, la filosofía surge de una experiencia fundamental. «La filosofía, como experiencia de la vida fáctica, requiere un motivo en el que permanezca la preocupación por la experiencia de la vida fáctica. Esto lo calificamos como la experiencia fundamental filosófica [philosophische Grunderfahrung] (esta es la confirmación de este motivo). No es ninguna iluminación especial, sino que es posible en cada existencia concreta donde la preocupación trae de vuelta a la existencia actual». ${ }^{52}$ Hay filosofía, hay cuestionamiento, porque en la misma vida se dan

50 SKS 7, Afsluttende Uvidenskabelig Efterskrift, p. 77.

51 «La separación entre una filosofía estrictamente científica y una filosofía de la concepción del mundo o -como Jaspers propone en su Psicología de las concepciones del mundo - entre una filosofía que meramente observa científicamente y una filosofía profética [prophetische Philosophie] no es del todo clara ni radical, precisamente porque no se pregunta si los dos fenómenos: "ciencia" y "concepción del mundo" puede ser relacionados con la idea de filosofía en primer lugar». GA 59, p. 11. Si tenemos en cuenta este análisis que Heidegger propone en su curso de 1920 Phänomenologie der Anschauung und des Ausdrucks respecto de la oposición propuesta por Jaspers entre una filosofía que observa y una filosofía que profetiza, no parece adecuado caracterizar la actitud filosófica heideggeriana como profética. Sin embargo, si se avanza en la lectura del curso, se advierte que los intentos por alcanzar un ámbito previo a la distinción misma se nutre preferentemente de elementos propios de la «filosofía profética». La labor de destrucción a la que Heidegger somete a ambas filosofías es más extrema en el caso de Natorp que en el caso de Dilthey. «Al contrario de la vacía filosofía formal trascendental, la filosofía de la vida es un paso necesario en el camino de la filosofía». Ib., p. 154. En Marburgo, Heidegger también se mostrará contrario al «profetismo filosófico»: «La fenomenología, mientras se entienda a sí misma, seguirá esa vía de investigación, contra cualquier tipo de profetismo, contra cualquier inclinación a hacer de guía de la vida». GA 20, p. 109. Pero esto no significa que su filosofía no pueda representar una «destrucción» del profetismo que lo devuelve a su verdadera dimensión: la de la comunicación indirecta, que mienta el profetismo del no profetizar.

52 GA 59, p. 174. Unos renglones más abajo, Heidegger destaca que «el único que está 
ciertas experiencias fundamentales reveladoras del carácter propio de nuestra existencia. Permaneciendo fiel a esta experiencia, el profeta profetiza, el hermeneuta interpreta, «destruye» la historia de ocultamiento ${ }^{53}$ y pone en evidencia la inadecuación de las lecturas tradicionales que obstruyen este tipo de experiencias en las que radica la posibilidad de una existencia propia. El profeta, en su anuncio del Mesías, asume una tarea interpretativa crítica pues cree en la posibilidad del cambio, cree en la posibilidad de la redención. La filosofía profética no acepta una labor meramente contemplativo-descriptiva, sino que busca intervenir en un mundo que ostensiblemente aun no ha sido redimido. Sin embargo, como en el caso de Kierkegaard, el profetismo de Heidegger pone en cuestión a la idea tradicional de profetismo. Ciertas convicciones que operan como supuestos los llevan a una revisión metodológica de la tarea profética. En el primer apartado, siguiendo la propuesta de Luis Guerrero, enuncié tres supuestos del pensamiento kierkegaardiano que le exigen adoptar una estrategia indirecta: la afirmación de una verdad subjetiva que se da en la apropiación, el hecho de la libertad y la existencia cristiana como existencia auténtica. Si bien no se puede afirmar sin más que Heidegger comparte estos supuestos, sí es posible considerar que existe cierta similitud en el talante «ético» de sus planteos. Está claro que el acercamiento fenomenológico-hermenéutico heideggeriano difiere del kierkegaardiano; pero entiendo que, a partir de un análisis de las consecuencias éticas de la indicación formal, es posible afirmar que ella da cuenta de una apropiación de los supuestos de Kierkegaard: la indicación formal da lugar a la afirmación de la posibilidad de una existencia auténtica que exige un particular respeto por la libertad basada en la afirmación del carácter decisivo de la apropiación individual que se da en el sentido de realización.

La tarea del profeta sólo puede ser reivindicada como comunicación indirecta. Jaspers define la comunicación indirecta en los siguientes términos: «Comunicación indirecta no significa que algo sea callado voluntariamente, que el hombre se ponga una máscara y, por principio, calle lo que ya sabe. Esto sería engaño o técnica pedagógica de un hombre superior. Comunicación indirecta significa que en los impulsos muy intensos de claridad y en la búsqueda total de formas y fórmulas no basta ninguna expresión y el hombre se hace consciente de ello; significa la actitud de que todo lo comunicado que existe directamente y que es decible, es, a fin de cuentas, lo inesencial pero, al mismo tiempo, portador indirecto de lo esencial. Ninguna doctrina es vida y ninguna comunicación de

en camino hacia esta filosofía, sin ver, sin embargo, su camino, es Jaspers (Psychologie der Weltanschauung). Y es sólo posible en base a las intuiciones diltheyanas». Id.

53 En los anexos de su último curso dictado en Friburgo Ontología. Hermenéutica de la facticidad se lee: «iHermenéutica es destrucción!». GA 63, p. 105. La hermenéutica ontológica heideggeriana implica una «destrucción" de las falsas concepciones filosóficas en las que estamos inmersos. 
una doctrina una transmisión de vida. La comunicación indirecta, es decir, la experiencia de lo directo como un médium en el que todavía actúa otro, es algo así como si aquí comunicara la vida misma. [...] Kierkegaard llama directamente a la comunicación indirecta, "comunicación existencial" [Existenzmitteilung]». ${ }^{54}$ Lo esencial, lo único importante a transmitir es esencialmente intransmisible. Esto es lo que Kierkegaard y Heidegger comprenden: el carácter inefable, en cada ocasión absolutamente individual, de la existencia que obliga a dejar de lado toda metodología directa. «Para él [el individuo] no existe la posibilidad de ser aconsejado por otro en lo esencial»». ${ }^{55} \mathrm{Y}$ es justamente en este punto donde radica el tenor ético de la metodología heideggeriana. Como Kierkegaard, el joven Heidegger es también un profeta de la comunicación indirecta, pues su intención, precisamente, es la de no convertirse en un profeta sino solamente estimular, llamar la atención, crear un estado de intranquilidad, volver las cosas problemáticas; pero sin dar prescripciones ni enseñar cómo vivir. ${ }^{56}$ Como bien destaca van Buren, las indicaciones formales son verdaderas preguntas formuladas de manera indirecta para que el otro investigue en su propia respuesta. ${ }^{57}$ No es posible suplantar al otro en esta tarea, no es posible darle reglas ni programas, lo único que podemos hacer es indicar formalmente lo que significa hacerse cargo de sus posibilidades. Este es el peculiar respeto que Heidegger demuestra por la libertad del otro y del que da cuenta la indicación formal. Libertad que, más allá de las diversas concepciones de la subjetividad a las que responde en Kierkegaard y en Heidegger, en ninguno de los dos casos puede ser entendida como arbitrio. En ambos autores, la libertad se limita a la posibilidad de asumir o no lo que ya se es, es decir, dar el salto de la fe asumiendo que somos «pecado» en términos de Kierkegaard, o asumir propiamente que somos «impropiedad» (Uneigentlichkeit) en términos de Heidegger. ${ }^{58}$ Ningún individuo puede ser sustituido en esta tarea. En este sentido, van Buren afirma que Heidegger previene a su lector de una interpretación de las indicaciones formales en el sentido de una comunicación directa: «él enfatiza, en el espíritu de la mayéutica socrático-kierkegaardiana, que las indicaciones deben alejar a los lectores individuales del contenido comunicativo directo de sus textos y del mens auctoris, y que deberían devolverlos a su propia actualización crítica de las guías hacia el tópico mismo, de tal manera que al final encuentren su propio camino». ${ }^{59}$

La comunicación indirecta kierkegaardiana pone al individuo en la difi-

54 K. Jaspers, Psychologie der Weltanschauungen, op. cit., p. 378.

55 Id.

56 Cf. Ib., p. 487.

57 J. van Buren, The young Heidegger, op. cit., p. 338.

58 GA 2, p. 238.

59 J. van Buren, The young Heidegger, op. cit., p. 339. 
cultad de encontrar la verdad en su propia existencia. La indicación formal heideggeriana opera de manera similar pues reenvía a la apropiación por parte del individuo desde su situación particular. Aprehender un fenómeno por medio de una indicación formal exige exceder toda predeterminación objetivante, pues su sentido último (sentido de realización) sólo puede manifestarse en la apropiación individual en cada caso. Es más, esta actualización de la indicación formal en el sentido de realización implica su renuncia a dar respuestas últimas, implica la necesidad del constante recomienzo de la filosofía, su estar siempre «en camino» que caracteriza su incesante preguntar. En este sentido, van Buren destaca que la indicación formal se constituye literalmente en una ética del méthodos, una ética del «estar en camino». La ética heideggeriana está contenida en su propuesta metodológica. La indicación formal permanece abierta al acontecer del fenómeno, se detiene ante él pues es el mismo fenómeno quién dicta su interpretación. James K.A. Smith ${ }^{60}$ insiste en que este aspecto de la metodología heideggeriana es también aplicable a nuestra relación con los otros; pues, si bien la indicación formal no está dirigida directamente al problema de la alteridad o de la trascendencia de una otredad ética, sí lo está de manera indirecta fundamental, pues la previsión metodológica de la indicación formal es la herramienta de la filosofía que impide predeterminar cualquier fenómeno. En este sentido, la crítica a la conceptualización teórica formulada por Lévinas en Totalidad e infinito no sólo no es aplicable al joven Heidegger, sino que se inscribe en la línea crítica por él inaugurada. Lévinas sostiene que el concepto aparece como un «tercer término» que se presenta como el «modo de privar al ser conocido de su alteridad». El objeto es privado de su trascendencia y forzado a presentarse en la esfera de lo Mismo según normas ya preestablecidas. ${ }^{61}$ La previsión levinasiana frente a la conceptualización teórica es similar a la de Heidegger, ambas se proponen impedir la reducción del fenómeno a los términos preestablecidos por la esfera de lo Mismo.

La indicación formal constituye una ética del método en tanto conmina a permanecer «en camino» sin poder reducir el darse del fenómeno mediante anticipaciones conceptuales que no respeten su carácter de alteridad. Si la filosofía es un cuestionamiento radical, el fenómeno no puede ser traído a la presencia en forma objetivada, sino que debe permanecer indicado provisionalmente en su alteridad, en su trascendencia.

60 Cf. J. K. A. Smith, Speech and Theology. Language and the Logic of Incarnation. London: Routledge, 2002, pp. 85-86.

61 Cf. E. Lévinas, Totalité et Infini. Essai sur l'extériorité. La Haye: Martinus Nihjoff, 1971, p. 34. 
Jorge Luis Roggero es miembro del Instituto de Filosofía “Alejandro Korn” de la Facultad de Filosofía y Letras de la Universidad de Buenos Aires.

Publicaciones recientes:

- Artículo: "Hingabe an die Sache. Misticismo y fenomenología en la ciencia originaria preteorética del joven Heidegger", Revista Latinoamericana de Filosofía, volumen 38, número 2 (primavera 2012), pp. 205-232. ISSN 0325-0725.

-Artículo: "Luther furieux", Revue Silène. Centre de Recherche en Littérature et Poétique comparées de l'Université de Paris X-Nanterre, Littérature et idée, revue en ligne, 18/11/2012. ISSN 2105-2816.

- Traducción y notas: M. Heidegger, "El problema del pecado en Lutero", Escritos de Filosofía. Revista de la Academia Nacional de Ciencias de Buenos Aires, segunda serie, año II, número 2 (2014), pp. 215-228. ISSN 2344-9586.

Línea de investigación: fenomenología y hermenéutica, filosofía de la religión, Martin Heidegger, Jean-Luc Marion, fenomenología francesa actual.

Dirección postal: Uriarte 2462, piso 6to departamento "B", CP 1425, Ciudad Autónoma de Buenos Aires, tel. 4779-9536.

E-mail: jorgeroggero@hotmail.com 
